\title{
Multi-assortment production flow synchronization. Multiscale modelling approach
}

\author{
Bozena Skolud $^{1, *}$, Damian Krenczyk ${ }^{1}$, and Reggie Davidrajuh ${ }^{2}$ \\ ${ }^{1}$ Silesian University of Technology, Gliwice, Poland \\ ${ }^{2}$ University of Stavanger, Stavanger, Norway
}

\begin{abstract}
Increasing competition strongly influence a company that is looking for the efficient method of production control. Companies expand their production flexibility and control method in the whole value chain. In this paper, authors propose a new description of the production inspired by the multiscale modelling. In particular, the idea based on the modelling of concurrent cyclic processes and the ability to use this approach in complex production systems is presented. This paper also discusses modelling and simulation of multiscale production flows using the Activity-Oriented Petri Nets.
\end{abstract}

\section{Introduction}

Success of every firm depends on effective utilisation of its resources (machines, finances, employee skills, etc.) Recently the competitive advantages, however, are not in the monthly, quarterly and annual aggregated level of production flexibility but on a daily and weekly basis on the market-driven flexibility [1]. This approach is observed in the automotive industry, which adapts flexible manufacturing systems. Firms continue to expand their flexibility in the whole value chain.

Enterprises producing a variety of products tend to stabilise production on the one hand, but on the other hand, must satisfy the expectation of demanding customers, which is characterized by high dynamics in today's market. In many cases, the production recognised by the customer as diversified and multi-variant, from the manufacturer point of view is seen as stable, because manufacturing of subsequent diverse products requires neither presenting nor other interventions. With the standardisation of certain parts (construction) transport, manufacturing, manipulation, the assembly can be carried out fluently. In many cases, final products are manufactured by methods characteristic for mass production. Suppliers of sub-elements and raw-materials for production have to adjust their production to recipient needs in term of assortment diversity, quantity, timeliness and delivery data (just in time or even just in sequence).

The objective of this paper is the presentation of approach, which could be considered as multiscale modelling of a production system. It is combination of multiscale idea known from material science and multi-assortment rhythmic production elaborated by authors of this paper in recent years.

* Corresponding author: bozena.skolud@polsl.pl 


\section{Multiscale in production}

There are phenomena in nature, which are observed in many scales. Such examples are atmospheric phenomena observed in the range of meters and on thousands of kilometres, and the chemical reactions that can last for seconds and hours. In chemistry, material science and mechanics a multiscale approach related to client expectation for material with specific properties is applied $[2,3,4]$.

Multiscale modelling involves the determination of material characteristics or behaviour of the system at one level by the use of information or a model from another level.

In relation to the production systems, the multiscale issue was presented in [5]. This approach is seen as linking production with sales. It shows the use of methods based on knowledge for effective modelling and simulation of assembly systems, so as to present the strong links between their infrastructure and products that are to be made in it. Therefore, it was created a library of resources based on knowledge, which is used for modelling at the line (cell) or at the level of the plant. The authors propose the use of the idea of multiscale modelling to control the flow of concurrent cyclic processes implemented at various levels of production (Fig. 1).

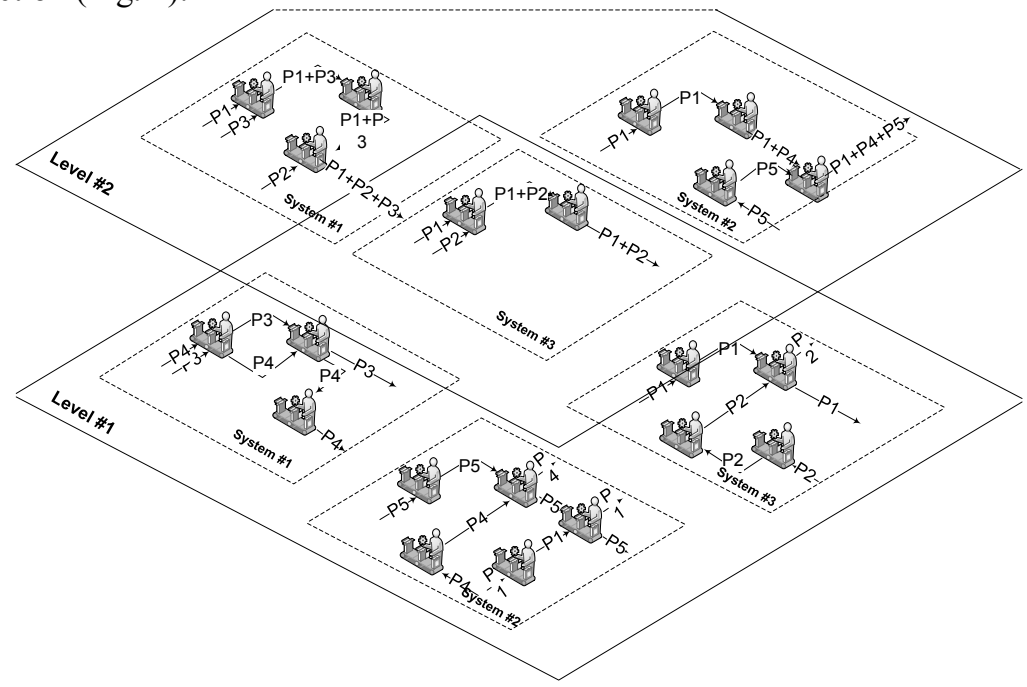

Fig. 1. Multiscale production system consist of multiple levels, where each level consists of many concurrent cyclic processes.

The proposed approach is associated with the control of production flow through the construction of a system of concurrent cyclic processes. This type of flow was chosen because for it at the planning stage an accurate evaluation of the functioning of the system is possible already.

It is assumed that sufficient condition guaranteeing deadlock-free and starvation-free process realisation in the system are satisfied. These conditions define a subset of the permitted dispatching rules controlling process execution.

This means, among other, that the sufficient conditions guaranteeing a cyclical process determined, provide the basis for an analytical determination of production flow parameters, such as:

- cycle (repetitiveness period) of the system,

- number of critical resources (resources fully occupied)

- storage capacity,

- timely realisation of production orders. 


\section{Rhythmic production modelling}

The problem of planning the production flow in production systems belonging to the class of Cyclic Concurrent Processes Systems (CCPS) is considered (Fig. 2). In the CCPS production systems, cyclic discrete processes $\mathrm{P}_{\mathrm{j}}$ are concurrently executed under mutual exclusions on the shared production resources $\mathrm{M}_{\mathrm{i}}$. The work cycle of the system is established by technological operations and the processes are sequentially carried out on the shared resource, in the order that is regulated with a local dispatching rules allocated on the resources $\mathrm{R}_{\mathrm{i}}$.

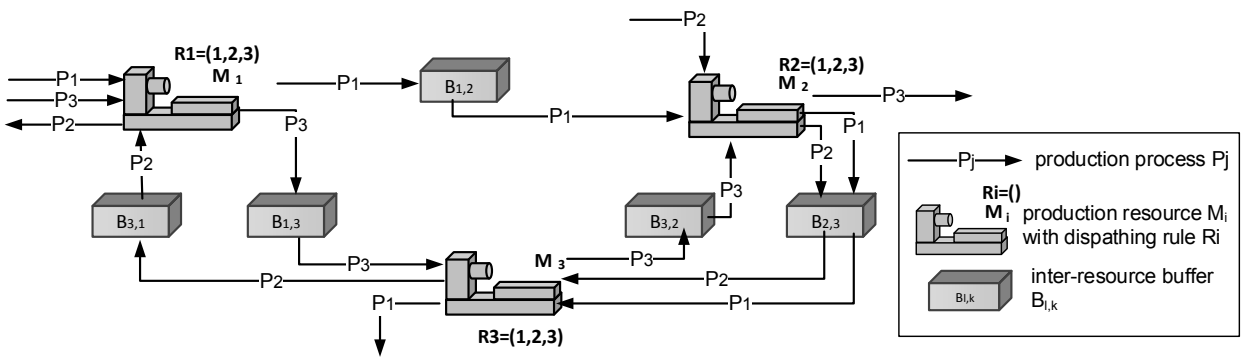

Fig. 2. System of concurrent processes.

The cyclic concurrent processes system with dispatching rules is defined as:

$$
S C_{l s}=\left(M_{l s}, P P, B, C s, R\right),
$$

where:

1 - level of production realisation,

$\mathrm{s}$ - production system ID,

$M_{l s}=\left\{M_{i}, i=1,2, \ldots, m_{l s}\right\}$ - the set of resources of s-th producer on 1-th prod. level,

$P P_{l s}=\left(P_{l s}, M P_{l s}\right)$ - the structure of the production processes of s-th producer on 1-th production level, where:

$P_{l s}=\left\{P_{j}, j=1,2, \ldots n_{l s}\right\}$ - the set of processes of s-th producer on 1-th level,

$M P_{l s}=\left\{M P_{j}, j=1,2, \ldots n_{l s}\right\}$ - the set of production processes matrix of s-th producer on 1-th production level:

$M P_{j}=\left[\begin{array}{cccccc}m p_{1,1} & m p_{1,2} & \ldots & m p_{1, h} & \ldots & m p_{1, H_{j}} \\ m p_{2,1} & m p_{2,2} & \ldots & m p_{2, h} & \ldots & m p_{2, H_{j}} \\ m p_{3,1} & m p_{3,2} & \ldots & m p_{3, h} & \ldots & m p_{3, H_{j}}\end{array}\right], j$-th production process matrix,

where:

$h$ - operation sequence number (according to the order of route operations),

$H_{j}$ - the number of operations in the $j$-th process route,

$m p_{l, h}$ - the resource number, on which the $h$-th operation is performed,

$m p_{2, h}$ - the cycle time of $h$-th operation,

$m p_{3, h}$ - the setup time of $h$-th operation,

$N$, - the batch size,

$T$ - the period of batch entering the system,

$B_{l s}=\left\{B_{u, k}, u=1,2, \ldots, m_{l s} ; k=1,2, \ldots, m_{l s} ; u \neq k\right\}$ - the set of inter-resource buffers

allocated to the neighbouring resources $\left(M_{u}, M_{k}\right)$,

$R_{l s}=\left\{R i, i=1,2, \ldots, m_{l s}\right)$ - the set of dispatching rules allocated on the resources.

In $[6,7]$ it has been proven that in the system when processes routes do not create closed loops a deadlock problem does not appear. 


\subsection{Balance condition}

A system of repetitive production is characterised by a simultaneous realisation of processes; this approach is not limited to production line. However, one should remember that in the case when production routes create a closed loop, the deadlock can appear. To avoid this problem sufficient condition concerning a flow balance and buffers capacity should be satisfied for deadlock free and starvation free processes realisation. The balance condition in the system is satisfied when number of elements entering the system in one repetitive period (common multiple of system cycle $T$ ) should be equal to the number of elements leaving the system in the same period. The system balance is accomplished in the case when the following equations are satisfied for the shared machine $[7,8,9]$ :

$$
\begin{gathered}
\chi_{1} n_{1,1}=\chi_{2} n_{2,1}=\ldots=\chi_{i} n_{i, 1}=\ldots=\chi_{m} n_{m, l}, \\
\ldots \\
\chi_{1} n_{1, j}=\chi_{2} n_{2, j}=\ldots=\chi_{i} n_{i, j}=\ldots=\chi_{m} n_{m, j}, \\
\ldots \\
\chi_{1} n_{1, n}=\chi_{2} n_{2, n}=\ldots=\chi_{i} n_{i, n}=\ldots=\chi_{m} n_{m, n},
\end{gathered}
$$

where:

$\chi_{i}$ - the repetitiveness of the dispatching rule allocated on the $i$-th machine,

$n_{i, j}$ - the repetitiveness of the $j$-th process in the dispatching rule allocated on the $i$-th machine.

Additionally the balance must be satisfied between all the levels of production. The cycle time for these levels can differ (on a common multiple). The balance is satisfied when (3) is satisfied:

$$
\sum_{s=1}^{S_{1}} \frac{T}{T_{1, s}} \chi_{i}^{1, s} n_{i, j}^{1, s}=\sum_{s=1}^{S_{2}} \frac{T}{T_{2, s}} \chi_{i}^{2, s} n_{i, j}^{2, s}=\cdots=\sum_{s=1}^{S_{l}} \frac{T}{T_{l, S}} \chi_{i}^{l, s} n_{i, j}^{l, s}=\cdots \sum_{s=1}^{S_{L}} \frac{T}{T_{L, S}} \chi_{i}^{L, s} n_{i, j}^{L, s}
$$

where:

$S_{l}-\quad$ No. of producer (production systems) on $l$-th level,

$\mathrm{T}$ - $\quad$ common multiplicity of cycles,

$T_{l, s}-\quad$ cycle time of $s$-th system on $l$-th level,

$\chi_{i}^{l, s}-\quad$ the repetitiveness of the dispatching rule allocated on the $i$-th machine of $s$ - $t h$ system on $l$-th level,

$n_{i, j}^{l, s}$ - the repetitiveness of the $j$-th process in the dispatching rule allocated on the $i$-th machine of $s$-th system on $l$-th level,

$L$ - No. of levels.

\subsection{Interoperation buffers allocation}

Buffers allocation and their capacities are one from list of constraints which should be satisfied in order to ensure deadlock free and starvation free functioning of the system. This ensures a bottleneck resources utilisation according to theory of constraints (TOC) investigated and described in [10]. Constraints in the production system are divided into two groups, i.e. the producers constraints and the customers constraints.

Simultaneously with the balance condition, the condition of the buffers space allocation in the system should be satisfied. It means that the capacity of buffer $B_{l, k}$ allocated between machines $M_{i}$, and $\mathrm{M}_{\mathrm{k}}$ for the $j$-th process should satisfy the following condition (4):

$$
B_{l . k}^{j}=n_{l, j} \cdot \chi_{l}
$$


Interoperation buffers capacity condition assures that capacity of buffer allocated between resources $M_{l}$, and $M_{k}$ should not be less than the number of elements executed according given process during one cycle (see equation (4)).

Interoperation buffers capacity condition must be satisfied also between levels (5)

$$
\mathrm{B}_{1 . \mathrm{k}}^{\mathrm{j}} \geq \frac{T}{T_{1, s}} \chi_{i}^{1, s} n_{i, j}^{1, s}
$$

This condition provides a storage capacity that guarantees preservation of all the elements made in the common multiple of the work period $(\mathrm{T})$ of the system.

\section{Modelling with GPenSIM}

Petri net model for a multiscale production flow can be obtained in three steps. Step-1: A system: a system within a level is modelled as a submodule (see fig.3a). Step-2: A level: the submodules that are representing the systems within a level are connected to form a module that represents a level of production flow, as shown in the fig. 3b. Step-3: Multi-level (multi-scale).

a)

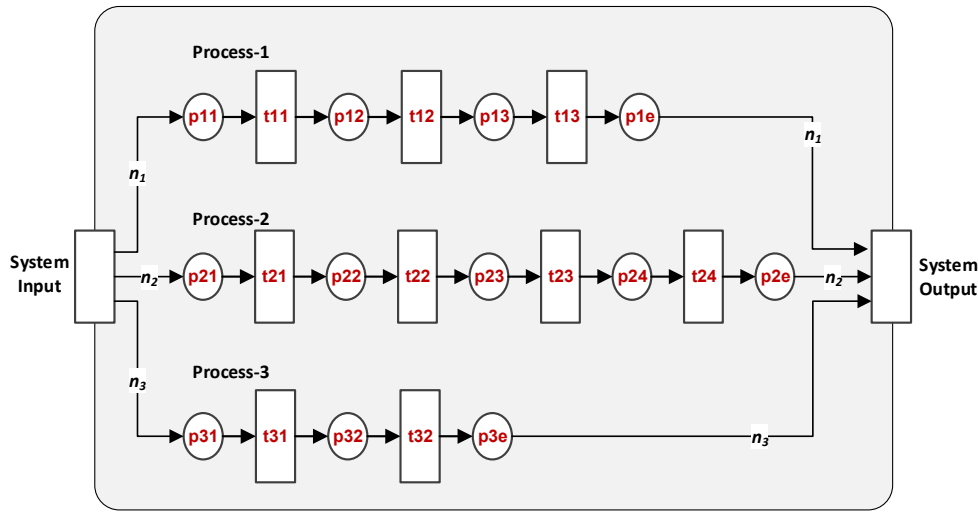

b)

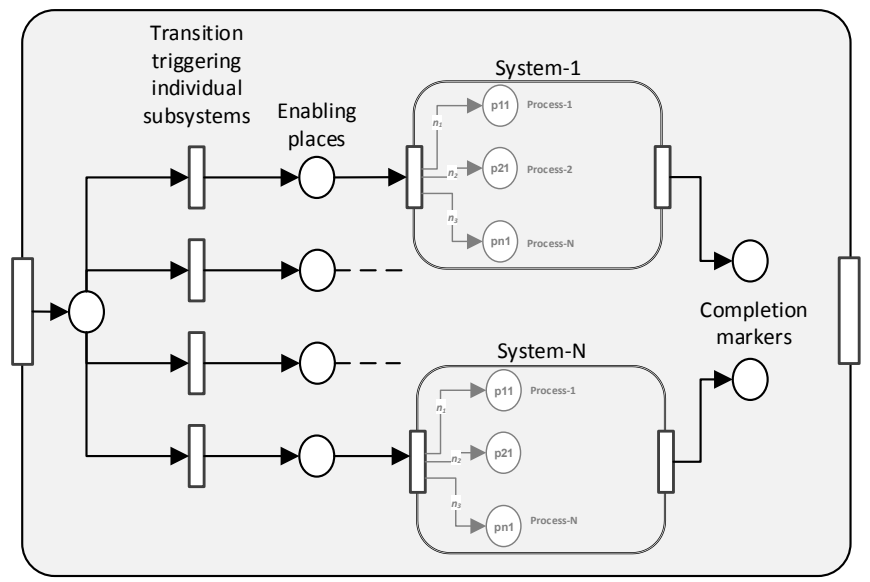

Fig. 3. a) The level module consisting of many system submodules; individual subsystems are enabled by the controlled firing of the triggering transitions; b) The system submodule, with three internal processes, each with 3, 4, and 2, operations, respectively. 
Finally, the modules that represent the levels are connected to form the complete model for the multiscale production flow.

It may seem that the resulting model will be huge. However, by compact modules obtained by the AOPN approach $[11,12]$ and with the support for modular Petri net model building provided by the GPenSIM software [13], very useful Petri net model can be obtained for multiscale production flow

\section{Conclusions}

In the paper, the approach to the rhythmic production planning inspired by multiscale modelling is presented. The production flow is controlled by dispatching rules, which are repetitive and are allocated to the resources. The approach proposed in the article is based on the previous work of the authors, which is used for the propagation of constraints. The proposed solution containing the multiscale model of the production system with the formulated sufficient conditions of processes flow will allow to obtain a solution in the form of dispatching rules for different levels, without the need for use of exhaustive search methods. They allow also immediate determination of selected qualitative and quantitative indicators values of the system.

The continuation of this approach is to look at the production through the context of multiscale modelling and create additional rules involving different levels of production. The further work in this field will be an extension of the methodology to another parameter and more complicated systems.

\section{References}

1. T. Tomino, I. J. Production Economics 118, pp. 375-386, (2009)

2. E. Weinan, B. Engquist, Z. Huang, Phys. Rev. B 67, 092101 (2003)

3. S.A. Hosseini, N. Shah, Interface Focus, 1(2), pp. 255-262. (2011)

4. I.S. Cole, C. Chu, M. Breedon, D.Winkler, 20th International Congress on Modelling and Simulation (Modsim2013), pp. 726-732, (2013)

5. M. Neumann, C. Constantinescu, E. Westkamper, Procedia CIRP, 3, pp. 406-411, (2012)

6. B. Skołud, Journal of Machine Engineering. 8(2), pp. 5-13 (2008)

7. D. Krenczyk, B. Skołud, Hybrid Artificial Intelligent Systems, Lecture Notes in Computer Science, 6679, pp. 397-404, (2011)

8. D. Krenczyk, B. Skołud, Applied Mechanics and Materials, 657, pp. 961-965, (2014)

9. D. Krenczyk, A. Dobrzanska-Danikiewicz, Journal of Materials Processing Technology. 164 pp. 1388-1394, (2005)

10. E. Goldratt, The Goal. A process of Ongoing Improvement, North River Press, Great Barrington, Mass, (1992)

11. R. Davidrajuh, 2012 IEEE International Conference on Systems, Man, and Cybernetics (SMC), pp. 1201-1206. (2012)

12. B. Skolud, D. Krenczyk, R. Davidrajuh, International Joint Conference SOCO'16CISIS'16-ICEUTE'16. ICEUTE 2016. Advances in Intelligent Systems and Computing, vol 527, pp. 397-407, (2017)

13. R. Davidrajuh, GPenSIM. A general purpose Petri net simulator for mathematical modeling and simulation of discrete-event systems in MATLAB, url: http://www. davidrajuh. net/gpensim. 